\title{
Semi-Passive Chemical Oxidation Schemes for the Long-Term Treatment of Contaminants
}

\author{
Frank Schwartz \\ Department of Geological Sciences \\ Ohio State University \\ Columbus, $\mathrm{OH} 43210$
}

\section{General Comments}

In situ chemical oxidation or ISCO schemes involve the addition of a chemical oxidant, such as potassium permanganate (KMnO4), which destroys chlorinated solvents like TCE in a straightforward reaction. Although ISCO is now regarded as a developing technology in an industrial sense, beyond active flushing schemes, there have been relatively limited investigations in how ISCO might be better used. Our previous study showed that $\mathrm{KMnO} 4$ flushing approaches often would be frustrated by the inability to control the delivery of the treatment fluid due to precipitation of low-permeability reaction by-product like $\mathrm{MnO} 2$ and other problems. It was therefore suggested that development of a new ISCO scheme that can provide both destruction efficiencies and plugging control would be required. The goal of our current study is to develop a scientific basis for the use of new semi-passive, well-based ISCO systems for treating chlorinated ethylene in groundwater. More specifically, our work examines the possibilities of developing a slowrelease $\mathrm{KMnO} 4$ scheme. This scheme could be operated in a semi-passive manner with periodic additions of the slow-release $\mathrm{KMnO} 4$ solids into well-delivery systems. To our knowledge, a system of this type has not been demonstrated. Our current scientific work is then concerned with how to manufacture the slowrelease $\mathrm{KMnO} 4$ solids, how the well systems can be designed, and how they interact with the flow systems to maximize spreading. To achieve these goals, development of numerical models to simulate solute transport coupled with NAPL dissolution and chemical reaction with the oxidant is required. There is also a need for ways to control the local precipitation of $\mathrm{MnO} 2$ that could cause plugging near the wells, or at least remove the plugging materials. Moreover, the likely extent of spreading of $\mathrm{KMnO} 4$ added by the wells must be understood. Finally, the spacing of wells will depend in part how far KMnO4 ends up spreading away from the treatment zone. Thus, a geochemical study as to the reaction kinetics involving $\mathrm{KMnO} 4$ and natural aquifer materials including carbonates, metals, and natural organic matter is also required. We feel that research of the type we are conducting with the current DOE grant is vital to advancing the remediation technology to the industrial phase.

\section{Results}

As of the first year of this 3-year project, we have performed a series of flow tank experiments and numerical simulations to investigate the problems related to the delivery of the permanganate from a welldelivery system to a plume. In addition, we have manufactured several types of slow-release $\mathrm{KMnO} 4$ solids and tested their controlled-release properties and spreading patterns in porous media through column and flow-tank experiments. Additional flow-tank experiments are being performed to assess the efficiency of slow-release KMnO4 scheme in the destruction of TCE plume. Primary results have been presented in the national conferences and as papers in peer-reviewed publications. 2-1.Permanganate Delivery from the Wells One new delivery scheme involves mixing the appropriate quantity of $\mathrm{KMnO} 4$ as a solid into the zone of DNAPL contamination. This scheme takes advantage of the tendency for reaction products to reduce the permeability of the treatment zone, which will control the dissolution of the solid and keep the MnO4- in contact with the DNAPL. What needs to be determined, however, is how various solid forms of $\mathrm{KMnO} 4$ behave in porous media and dissolve under the combined influence of fluid flow and $\mathrm{MnO} 2$ precipitation. In order to effectively deliver permanganate solid to a contaminant plume, a well delivery system was studied as a proof-of-concept. The design includes a series of vertical wells to deliver solid $\mathrm{KMnO} 4$ into the subsurface by diffusion out of the well casing. The idea is to form a zone of localized reaction through dispersive mixing with dissolved TCE. Thus, as the contaminant plume passes the well system, in situ oxidation will destroy the contaminant and prevent further spreading. The well-delivery system uses the solubility of $\mathrm{MnO} 4$ - in water and dispersion to add appropriate quantities into the flow system. A thin, small 2-D glass flow tank with Teflon end fittings was constructed for the experiments. The 
flow tank was filled with medium silica sand. A $1 \mathrm{~mm}$ (inside diameter) Teflon tube was installed to represent wells. The experiment began by flowing de-ionized water through the tank for two days. The inlet pump was then switched to deliver dissolved TCE at a concentration of 25,000 $\mu \mathrm{g} / \mathrm{L}$. At the outlet, effluent samples were collected at regular time intervals. The narrow tank permitted back lighting and visual monitoring of the Mn oxide, which formed an observable brown precipitate. This experiment demonstrated that the well-delivery system could release MnO4- to the TCE plume at a stable, constant, and controllable rate. We have conducted numerical simulation to investigate the PRBS designed as a semi-passive permanganate delivery system - permanganate reactive barrier system (PRBS). Numerical simulation was conducted to elucidate the parameters that will influence the field implementation of a PRBS. SUTRA, a three-dimensional model for saturated-unsaturated, variable-density ground-water flow with solute or energy transport developed by USGS was used for the modeling. We investigated issues such as permanganate consumption by aquifer materials, variable density flow effect, as well as lateral spreading under different geological settings. Permanganate consumption rates determined from the batch experiment were used in the model. Our simulation has shown that density-driven flow can result in sinking of the permanganate plume and a deviation from a perfect match with the contaminant plume. In this case, a maximum target concentration for permanganate should be determined through modeling to avoid such a deviation. The knowledge gained from the numerical simulation will help to optimize the parameters for designing a PRBS at a remediation site. 2-2 Development of Slow-release KMnO4 Scheme 2-2-1 Manufacturing Slow-release $\mathrm{KMnO} 4$ As described earlier, the slow-release $\mathrm{KMnO} 4$ scheme takes advantage of the tendency for low permeability materials to control the dissolution of the solid KMnO4. To determine how $\mathrm{KMnO} 4$ solids dissolve in porous media under the combined influence of fluid flow and low-permeability materials, we monitored the effect of $\mathrm{MnO} 2$ formation in the porous media to $\mathrm{KMnO} 4$ dissolution and MnO4- release. Experimental conditions were provided by glass columns with porous media comprised sodium-thiosulfate (Na2S2O3), $\mathrm{KMnO} 4$, and silica sands. As a reductant, $\mathrm{Na2S} 2 \mathrm{O} 3$ reacts with $8 \mathrm{MnO} 2+$ permanganate to produce $\mathrm{MnO} 2$ precipitates: $8 \mathrm{MnO} 4-+\mathrm{H} 2 \mathrm{O}+3 \mathrm{~S} 2 \mathrm{O} 32-$ 6SO42- + 2OH- A geochemical equilibrium model calculation (PHREEQC, Parkhurst and Appelo, 1999) also showed that among the possible forms of manganese oxides, i.e. $\mathrm{MnO}$, $\mathrm{Mn3O} 4 \mathrm{MnOOH}$, or $\mathrm{Mn}(\mathrm{OH}) 2, \mathrm{MnO} 2$ is the favorable form of solid precipitates when thiosulfate is used as the reducing agent. Column experiments showed that the column with sand/KMnO4 mixture stopped discharging permanganate solution in about 70 minutes while the column with sands/KMnO4/Na2S2O3 mixture continued to discharge permanganate solution until after 270 minutes. Black $\mathrm{MnO} 2$ precipitates and purple permanganate solution were identified in the column with sands/KMnO4/Na2S2O3 mixture throughout the flushing period. This observation demonstrated that low-permeability material can substantially delay dissolution rate of $\mathrm{KMnO} 4$ solids in saturated porous medium. Based on the results from the dissolution tests, we have manufactured two types of slow-release $\mathrm{KMnO} 4$ products using different methods and materials. The products were prepared using cohesive, low-permeability materials that are inert to water and chlorinated solvents in low-temperature aqueous conditions as the matrix materials. $\mathrm{KMnO} 4$ grains of different size were then mixed with the cohesive materials at different ratios using a specially manufactured stainless-steel molding system. Chemicals known to dissolve or suppress $\mathrm{MnO} 2$ precipitates were also added to the mixtures to control plugging of the matrix. A total of 220 slow-release $\mathrm{KMnO} 4$ products have been manufactured for experimental use. 2-2-2 Estimating Release Rates of the Slow-release KMnO4 The controlled-release properties of the slow-release $\mathrm{KMnO} 4$ were tested using column experiments. Column tests demonstrated that the slow-release $\mathrm{KMnO} 4$ releases permanganate in a controlled fashion. The release rates were constrained by flow rates, size of the $\mathrm{KMnO} 4$ grains, mixing ratios of $\mathrm{KMnO} 4$ and the cohesive materials in the matrix, and thickness of the slow-release $\mathrm{KMnO} 4$. We measured permanganate concentrations of the column discharge throughout the testing period to estimate the temporal variations in the release rate. Permanganate concentrations were initially high at $24 \mathrm{mg} / \mathrm{L}$ on day 1 , then gradually decreased and stabilized at approximately $3 \mathrm{mg} / \mathrm{L}$ in four days. Permanganate concentrations then remained constant throughout the remainder of column experiments. Mean release rate was estimated as $55 \mathrm{mg} / \mathrm{day}$ and the life of the slow-release $\mathrm{KMnO} 4$ media was estimated as 640 days. 2-2-3 Flow Tank Experiment With the promising results from release rate tests, a flow-tank experiment was performed to estimate release and spreading pattern of the slow-release $\mathrm{KMnO} 4$ in sandy porous medium. A permeable reactive barrier system comprising two vertical lines of slow-release $\mathrm{KMnO} 4$ pellets was constructed in the upstream end of the flow tank. Permanganate concentrations remained constant throughout the flushing period of 93 days and ranged from $\sim 900 \mathrm{ppb}$ nearby the upstream PRB to $\sim 100 \mathrm{ppb}$ in the downstream end of the tank. Flow tank experiment demonstrated that our slow-release $\mathrm{KMnO} 4$ scheme releases 
permanganate a long-term, controlled manner. A flow-tank experiment was performed to estimate the efficiency of the slow-release KMnO4 scheme in the destruction of dissolved TCE present as a plume. The flow tank with constructed PRB in the upstream end was flushed with TCE contaminated water $(3.5 \mathrm{mg} / \mathrm{L})$ for 63 days. Permanganate and TCE concentrations were measured across the flow tank. TCE concentration remained below detection limit $(5 \mathrm{ppb})$ throughout the flushing period. Permanganate concentrations were in the range of 10 to $70 \mathrm{mg} / \mathrm{L}$ on day 5, then decreased to 10 to $30 \mathrm{mg} / \mathrm{L}$ range and remained constant throughout the testing period. The results of the flow tank experiment demonstrated that our slow-release $\mathrm{KMnO} 4$ scheme would be capable of controlling the low-concentration DNAPL plumes in a long-term, semi-passive manner. 